\title{
RECURSOS DIDÁTICOS EM TABLETS PARA O ESTUDO DE ÁREAS DE FIGURAS PLANAS: AVALIAÇÃO DA EFICÁCIA
}

\author{
Larissa de Sousa Moreira - IFFluminense Campus Campos Guarus \\ larissasm@iff.edu.br \\ Silvia Cristina Freitas Batista - IFFluminense Campus Campos Centro \\ silviac@iff.edu.br \\ Eduardo Shimoda - Universidade Cândido Mendes - Campos dos Goytacazes \\ shimoda@ucam-campos.br
}

\section{RESUMO}

O presente trabalho tem por objetivo analisar a avaliação da eficácia de um conjunto de applets GeoGebra para tablets, utilizados por meio de atividades investigativas, na aprendizagem de áreas de figuras planas, no Ensino Médio. Para tanto, foi realizado um experimento com três grupos de alunos. Dois desses sofreram intervenções com o uso dos applets e do livro didático e o terceiro foi um grupo de controle. Os dados, obtidos por meio de testes somativos, foram analisados estatisticamente. No que se refere à utilização das intervenções para aprendizagem, de forma geral, os applets mostraram-se significativamente eficazes com ou sem o uso do livro didático, sendo os resultados melhores quando foram utilizados antes do livro. Verificou-se também que os applets foram eficazes nos diferentes graus de dificuldade das questões do teste somativo.

Palavras-chave: applets GeoGebra, tablets, eficácia

\section{DIDACTIC RESOURCES FOR TABLETS IN THE STUDY OF PLANE FIGURES: EFFECTIVENESS EVALUATION}

\begin{abstract}
The purpose of this research is analyze the effectiveness of a set of GeoGebra applets for tablets, used through investigative activities, in the study of plane figures, in High School. Therefore, an experiment was carried out with three groups of students. Two of these groups have suffered interventions by the use of applets and the textbook and the third one was the control group. The data collected through summative assessment was statistically analyzed. Regarding the learning interventions, in general, the applets have been proved to be significantly effective with or without the use of the textbook, with best results achieved when the applets were used before the textbook. It was also possible to verify that applets were effective in questions with different levels of difficulty during the summative assessment.
\end{abstract}

Keywords: GeoGebra Applets, Tablets, Effectiveness

\section{Introdução}

$\mathrm{Na}$ Matemática, os tablets permitem a exploração de diferentes abordagens nas tarefas de representação de soluções, facilitam a visualização e a comunicação, favorecem o desenvolvimento do pensamento crítico, aumentam o interesse nos estudos e são de fácil aceitação (Amirnudin; Sulaiman, 2013; Riconscente, 2013; Agostinho, 2015).

Nesse sentido, diversas pesquisas têm investigado o uso de aplicativos educacionais em tablets na Matemática (Barcelos et al., 2013; Riconscente, 2013; 
Pitchford, 2015; Zhang et al., 2015). Dentre as vantagens, destacam-se a facilidade de visualização em 3D, o fornecimento de feedback imediato e o apoio ao desenvolvimento de habilidades.

Um tipo de aplicativo que pode ser utilizado em tablets é o applet GeoGebra, que é uma construção elaborada no GeoGebra, um software de Matemática Dinâmica. Um applet permite a visualização de vários elementos de forma dinâmica, no qual alguns objetos podem ser modificados enquanto outros são mantidos (Havelková, 2013).

Nesse contexto, o objetivo deste trabalho é analisar a avaliação da eficácia de um conjunto de applets GeoGebra para tablets, utilizados por meio de atividades investigativas, na aprendizagem de áreas de figuras planas, no Ensino Médio. Para tanto, foram desenvolvidos os applets e as atividades. A eficácia foi analisada por meio de testes estatísticos.

Tendo em vista esse objetivo, este artigo foi estruturado em quatro seções, além desta introdução. Na seção 2, são descritos trabalhos sobre avaliação da eficácia de aplicativos de Matemática para tablets. Na seção 3, apresentam-se os procedimentos metodológicos adotados e, na seção 4, discutem-se resultados encontrados. Finalizando, na seção 5, são apresentadas considerações sobre o estudo promovido.

\section{Avaliação da eficácia de aplicativos de Matemática para tablets}

$\mathrm{Na}$ área educacional, a eficácia de um recurso é alcançada quando os objetivos são atingidos, os resultados obtidos no processo de ensino e aprendizagem são satisfatórios e quando as necessidades da sociedade, em geral, e do aluno, em particular, são atendidas (Pereira; Peixe; Staron, 2010).

Segundo Adams e Chung (2013), há um excesso de estudos qualitativos que, muitas vezes, não trazem conclusões claras em relação à eficácia de aplicativos em tablets na melhoria da compreensão, colaboração e interação do aluno. Nesta seção, descrevem-se, brevemente, três estudos que analisam, quantitativamente, a eficácia de aplicativos de Matemática para tablets.

Riconscente (2013) investigou se o Motion Math, um jogo para iPad, aumenta o conhecimento sobre frações entre crianças. A autora dividiu 122 crianças da $5^{\mathrm{a}}$ serie, de duas escolas públicas da Califórnia, em dois grupos. Para o primeiro semestre do estudo, um grupo serviu como tratamento e o outro como controle e, no meio do estudo, os dois grupos trocaram. O tratamento consistiu em jogar Motion Math diariamente por 20 minutos, durante cinco dias letivos consecutivos, e o grupo controle teve instrução matemática regular sem envolver frações. Foram promovidos testes estatísticos para comparar um grupo com outro, ao longo do tempo, e as alterações do índice de acerto em cada grupo. Os resultados revelaram que, nas duas escolas, os ganhos significativos de aprendizagem dependiam do período em que os participantes jogavam Motion Math.

O estudo quantitativo de Pitchford (2015) avaliou a eficácia de uma intervenção baseada em tablet para apoiar habilidades matemáticas. Participaram da pesquisa 318 crianças de uma escola primária do Malawi, na África Oriental. Elas foram divididas, em três grupos: um que sofreu uma intervenção matemática por meio de tablet (grupo de tratamento), um que sofreu uma intervenção por meio de tablets, mas não estudou Matemática (grupo placebo), e um grupo que teve aulas tradicionais de Matemática (grupo controle). As crianças realizaram um pré-teste antes do experimento e um pósteste depois de uma intervenção de oito semanas. A intervenção do grupo de tratamento consistiu no uso de quatro aplicativos para o estudo de Matemática: Masamu 1, Masamu 2, Count to 10 e Count to 20. Na intervenção do grupo placebo, foram utilizados aplicativos educativos, mas que não envolviam conceitos matemáticos e isso buscou identificar possíveis efeitos, no uso de aplicativos matemáticos, de variáveis 
externas associadas com o uso de tablets. Os resultados indicaram que a intervenção com o uso de tablets pode adicionar um valor significativo à prática de sala de aula $\mathrm{e}$ apoiar o desenvolvimento de habilidades matemáticas de crianças da escola primária.

O estudo de Zhang et al. (2015) teve por objetivo investigar se determinados aplicativos de Matemática podem melhorar o rendimento de alunos com dificuldades de aprendizagem. Participaram da pesquisa 18 alunos do $4^{\circ}$ ano, com idade média de nove anos, de uma escola pública dos Estados Unidos. Foram utilizados os aplicativos Splash Math, Motion Math Zoom e Long Multiplication. Para medir a aprendizagem, os alunos realizaram três avaliações. As porcentagens de acerto em cada teste foram calculadas e analisadas estatisticamente. Os autores concluíram que o uso de aplicativos de Matemática pode ser uma prática eficaz no auxílio ao ensino de alunos com dificuldades.

Em geral, os aplicativos avaliados nos estudos apresentados foram eficazes. Também no presente trabalho buscou-se avaliar a eficácia de aplicativos para tablets, mas, diferentemente dos estudos mencionados, foram analisados applets GeoGebra e o público-alvo foi composto por alunos do Ensino Médio.

\section{Material e métodos}

Para a realização da pesquisa, foram desenvolvidos treze applets GeoGebra que têm os seguintes objetivos: i) possibilitar a compreensão de que figuras diferentes podem ter áreas iguais; ii) permitir verificar, geometricamente, que triângulos com bases iguais e alturas iguais possuem a mesma área; iii) permitir a dedução das fórmulas para o cálculo de áreas do quadrado, retângulo, paralelogramo, triângulo qualquer, triângulo equilátero, hexágono regular, trapézio, losango e círculo.

Além disso, foi elaborada uma apostila de atividades investigativas, intitulada "Estudando Áreas de Figuras Planas com applets GeoGebra em tablet". As nove atividades dessa apostila têm uma abordagem diferente dos exercícios tradicionais dos livros didáticos e foram desenvolvidas para serem resolvidas com o uso dos applets. Ao longo do texto, quando os applets GeoGebra forem mencionados, deve-se sempre considerar que o uso desses ocorreu por meio dessas atividades.

Participaram da pesquisa 56 alunos, de três turmas, matriculados no $1^{\circ}$ ano do Ensino Técnico integrado ao Ensino Médio de uma instituição pública federal do Noroeste do Estado do Rio de Janeiro. Todos já tinham experiência com tablets na aprendizagem de Matemática, com applets GeoGebra e já tinham estudado o tema áreas de figuras planas em algum momento da vida escolar. As três turmas possuíam a mesma professora de Matemática (primeira autora deste artigo) e esta atuou como intervencionista em todas as etapas. A pesquisa começou em novembro de 2015 e durou cinco semanas. Para avaliar a eficácia do uso dos applets, foram definidos três tipos de intervenção pedagógica:

- Intervenção L: com o uso de livro didático, abordando o tema áreas de figuras planas;

- Intervenção A: com o uso dos applets GeoGebra sobre áreas de figuras planas;

- Intervenção Controle: sobre um tema matemático diferente de áreas de figuras planas.

O livro didático utilizado durante o tratamento L foi o mesmo adotado nas turmas, na época da pesquisa.

O experimento foi realizado com três grupos de alunos, nomeados Grupo L-A, Grupo A-L e Grupo C. O Grupo L-A, com 22 alunos, sofreu a intervenção L e, em seguida, a intervenção A. O Grupo A-L, com 16 alunos, teve as intervenções na ordem contrária. O Grupo Controle, com 18 alunos, sofreu uma intervenção sobre conteúdo matemático diferente de áreas de figuras planas. As etapas executadas nos três grupos do experimento estão representadas na Figura 1. 
Um teste somativo com 12 questões objetivas sobre áreas de figuras planas foi elaborado para a coleta de dados. As questões desse teste estão de acordo com problemas disponíveis em livros didáticos e utilizados em vestibulares e cada uma delas apresenta quatro opções de resposta.

Todos os grupos realizaram esse teste três vezes, no início (Teste 1), no meio (Teste 2) e no fim do experimento (Teste 3). Todas as 12 questões foram utilizadas nas três versões do teste. No entanto, em cada uma das versões, elas apareciam em uma ordem diferente, bem como as opções.

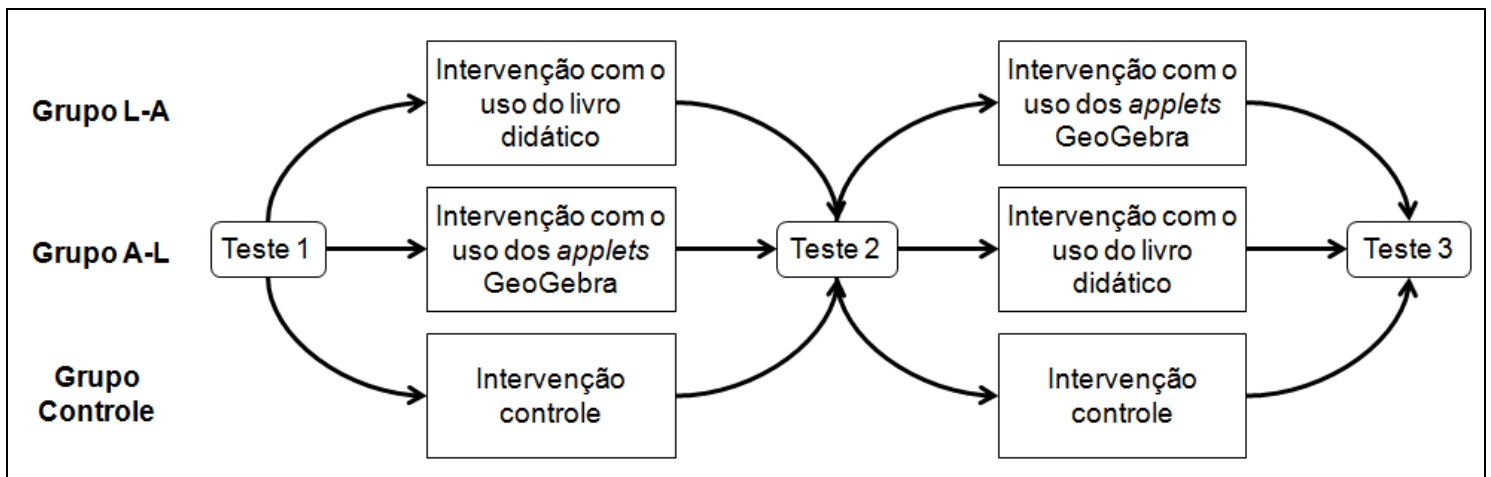

Figura 1 - Etapas do experimento

O Grupo Controle realizou os testes sem sofrer uma intervenção sobre áreas de figuras planas com o objetivo de identificar se a repetição dos testes iria influenciar no índice de acerto dos alunos.

A intervenção com livro didático ocorreu em uma sala de aula convencional, com o uso do quadro branco e marcadores coloridos. Os alunos estavam organizados em filas, cada um de posse de seu próprio livro.

A intervenção por meio dos applets GeoGebra ocorreu em uma sala de aula equipada com tablets Android e iPads. Como os applets podem ser acessados de qualquer dispositivo e por entender que não há diferença técnica que possa interferir de forma significativa no uso dos applets, optou-se por deixar que cada aluno escolhesse entre tablets Android e iPads.

A seguir, são descritos os cálculos e testes estatísticos efetuados. Os dados obtidos por meios desses são descritos e analisados na seção 4 .

\subsection{Cálculos para verificar a homogeneidade dos grupos e o efeito da repetição}

Para validar o experimento foi preciso realizar os testes estatísticos descritos no Quadro 1. A Análise de Variância (ANOVA) é utilizada para verificar se três ou mais médias são iguais.

Quadro 1 - Testes estatísticos realizados para validar o experimento

\begin{tabular}{|c|c|c|c|}
\hline \multicolumn{2}{|c|}{ "Composição do estrato } & \multirow{2}{*}{ Objetivo } & \multirow{2}{*}{$\begin{array}{c}\text { Teste } \\
\text { estatístico }\end{array}$} \\
\hline Teste & Grupo & & \\
\hline Teste 1 & $\mathrm{~L}-\mathrm{A}$ & \multirow{3}{*}{ Verificar a homogeneidade entre os grupos. } & \multirow{3}{*}{ ANOVA } \\
\hline Teste 1 & A-L & & \\
\hline Teste 1 & $\mathrm{C}$ & & \\
\hline Testes 1,2 e 3 & $\mathrm{C}$ & Verificar o efeito da repetição dos testes na média de acerto. & ANOVA \\
\hline
\end{tabular}

\subsection{Cálculo das médias dos efeitos do livro didático e dos applets}

Para analisar os efeitos das intervenções, as médias de acertos depois de cada uma delas foram calculadas e comparadas conforme o Quadro 2. O Teste t é utilizado para avaliar se há diferença significativa entre as médias de duas amostras. 
Quadro 2 - Testes estatísticos para verificar o efeito do livro e dos applets

\begin{tabular}{|c|c|c|c|c|}
\hline \multirow{2}{*}{ Fonte de variação } & \multirow{2}{*}{$\begin{array}{c}\text { Estrato } \\
\text { comparado }\end{array}$} & \multicolumn{2}{|c|}{ Composição do estrato } & \multirow{2}{*}{$\begin{array}{c}\text { Teste } \\
\text { estatístico }\end{array}$} \\
\hline & & Testes & Grupo & \\
\hline \multirow{5}{*}{ Livro } & \multirow{3}{*}{ Sem livro } & 1,2 e 3 & $\mathrm{C}$ & \multirow{5}{*}{ Teste $\mathrm{t}$} \\
\hline & & 1 e 2 & A-L & \\
\hline & & 1 & L-A & \\
\hline & \multirow{2}{*}{ Com livro } & 2 e 3 & L-A & \\
\hline & & 3 & A-L & \\
\hline \multirow{5}{*}{ Applets } & \multirow{3}{*}{ Sem applets } & $1,2 \mathrm{e} 3$ & $\mathrm{C}$ & \multirow{5}{*}{ Teste $\mathrm{t}$} \\
\hline & & 1 e 2 & L-A & \\
\hline & & 1 & A-L & \\
\hline & \multirow{2}{*}{ Com applets } & 2 e 3 & A-L & \\
\hline & & 3 & L-A & \\
\hline
\end{tabular}

\subsection{Cálculo do nível de dificuldade das questões}

Para classificar as questões por nível de dificuldade, calculou-se a média de acerto de cada uma das 12 questões do Teste 1 de todos os grupos, utilizando a fórmula apresentada na Figura 2.

$$
\text { Média de acerto da questã̃o }=\frac{\mathrm{n}^{0} \text { de acertos da questẫo }}{\mathrm{n}^{0} \text { total de participantes }} \times 100
$$

Figura 2 - Fórmula para o cálculo da média de acerto no Teste 1

As quatro questões com os maiores índices de acerto foram classificadas como fáceis, as quatro com os menores, como difíceis e as outras quatro como médias. A média de acerto de cada nível de questão foi calculada utilizando a fórmula da Figura 3. Média de acerto em cada nível $=\frac{\sum \text { médias de acerto das questões de mesmo nível }}{4} \times 100$

Figura 3 - Fórmula para o cálculo da média de acerto de cada nível de questão

\subsection{Cálculo do efeito da ordem de uso dos applets}

Para identificar se houve diferença significativa entre as médias finais dos grupos que tiveram a intervenção dos applets em momentos diferentes, foram utilizadas as médias do Teste 3 dos Grupos L-A e A-L (Quadro 3). Novamente, utilizou-se o Teste t.

Quadro 3 - Comparação realizada entre as notas finais dos dois grupos de tratamento

\begin{tabular}{|c|c|c|c|c|c|}
\hline \multirow{2}{*}{$\begin{array}{l}\text { Fonte de } \\
\text { variação }\end{array}$} & \multirow{2}{*}{$\begin{array}{c}\text { Estratos } \\
\text { comparados }\end{array}$} & \multicolumn{2}{|c|}{$\begin{array}{c}\text { Composição do } \\
\text { estrato }\end{array}$} & \multirow[t]{2}{*}{ Objetivo } & \multirow{2}{*}{$\begin{array}{c}\text { Teste } \\
\text { estatístico }\end{array}$} \\
\hline & & Teste & Grupo & & \\
\hline \multirow{2}{*}{$\begin{array}{l}\text { Ordem das } \\
\text { intervenções }\end{array}$} & $\begin{array}{l}\text { Livro antes } \\
\text { dos applets }\end{array}$ & $\begin{array}{c}\text { Teste } \\
3\end{array}$ & L-A & \multirow{2}{*}{$\begin{array}{l}\text { Verificar se houve diferença } \\
\text { significativa entre os grupos que tiveram } \\
\text { a intervenção dos } \text { applets em momentos } \\
\text { diferentes. }\end{array}$} & \multirow{2}{*}{ Teste $\mathrm{t}$} \\
\hline & $\begin{array}{l}\text { Applets antes } \\
\text { do livro }\end{array}$ & $\begin{array}{c}\text { Teste } \\
3\end{array}$ & A-L & & \\
\hline
\end{tabular}

\subsection{Cálculo do efeito dos applets nos estratos}

Para analisar o efeito do uso dos applets quando utilizados sozinhos e antecedidos ou precedidos pelo uso do livro didático, as médias referentes a cada intervenção foram calculadas, conforme o Quadro 4.

Quadro 4 - Relação das médias coletadas

\begin{tabular}{|c|c|c|}
\hline \multirow{2}{*}{ Estratos comparados } & \multicolumn{2}{|c|}{ Composição dos estratos } \\
\cline { 2 - 3 } & Teste & Grupos \\
\hline \multirow{2}{*}{ Controle } & 1 & L-A e A-L \\
\cline { 2 - 3 } & 1,2 e 3 & C \\
\hline Somente Applets & 2 & A-L \\
\hline Somente Livro & 2 & L-A \\
\hline Applets e Livro & 3 & L-A e A-L \\
\hline
\end{tabular}


Para verificar se houve diferença significativa entre as intervenções, foi realizada a ANOVA entre as médias obtidas. As médias referentes a cada intervenção também foram comparadas dentro de cada nível de questão. Para tanto, foi realizada a ANOVA e o Teste de Tukey. O Teste de Tukey é aplicado quando a ANOVA é significativa e testa as diferenças entre duas médias para identificar qual(is) tratamento(s) é (são) diferente(s).

Os resultados encontrados são apresentados na seção a seguir.

\section{Resultados e discussão}

A aplicação do Teste 1 nos três grupos ocorreu na primeira semana e durou cerca de 50 minutos em cada grupo. Na segunda semana, o Grupo L-A sofreu a intervenção por meio do livro didático e o Grupo A-L, por meio dos applets GeoGebra. As duas intervenções duraram 100 minutos cada uma.

O Teste 2 foi aplicado nos três grupos na terceira semana e, como o teste 1, durou 50 minutos. Na quarta semana, o Grupo L-A sofreu a intervenção com os applets e o Grupo A-L, com o livro didático, e cada uma durou 100 minutos. Por fim, na quinta semana, os três grupos realizaram o Teste 3 , também em 50 minutos.

A maioria dos participantes completou todas as etapas do experimento. Sete participantes estiveram ausentes em pelo menos uma etapa da pesquisa e tiveram os dados excluídos na análise. A Tabela 1 apresenta os grupos, o número de alunos total e o número de dados analisados.

Tabela 1 - Total de alunos por turma e número de participantes

\begin{tabular}{lcc}
\hline Grupo & $\mathrm{N}^{\circ}$ de alunos na turma & $\mathrm{N}^{\circ}$ de alunos com dados analisados \\
\hline L - A & 22 & 19 \\
A - L & 16 & 14 \\
Controle & 18 & 16 \\
\hline
\end{tabular}

\subsection{Homogeneidade dos grupos e efeito da repetição dos testes}

A Tabela 2 mostra as médias obtidas em cada um dos testes pelos três grupos.

Tabela 2 - Médias obtidas em cada teste pelos grupos

\begin{tabular}{lccc}
\hline Grupo & Teste 1 & Teste 2 & Teste 3 \\
\hline L - A & $44,4 \%$ & $42,7 \%$ & $61,4 \%$ \\
A - L & $47,4 \%$ & $59,5 \%$ & $74,0 \%$ \\
Controle & $52,8 \%$ & $54,2 \%$ & $44,4 \%$ \\
\hline
\end{tabular}

As análises estatísticas das médias de acerto de cada grupo no Teste 1 não revelaram diferença significativas $(\mathrm{P}=0,1208)$. Assim, é possível afirmar que os três grupos são homogêneos.

Além disso, verificou-se que não houve diferença significativa entre as médias dos Testes 1,2 e 3 do Grupo Controle $(P=0,0985)$. Assim, estatisticamente, a repetição do conjunto de questões em três momentos diferentes, em um intervalo de 15 dias entre eles, não influenciou o índice de acerto dos alunos do Grupo Controle.

Sendo os três grupos homogêneos, é possível afirmar que, estatisticamente, a repetição dos testes também não influenciou a média de acerto dos Grupos L-A e A-L.

\subsection{Efeitos do livro didático e dos applets}

Sobre o efeito do uso livro didático, verificou-se que o índice de acerto dos alunos sem o livro foi de $49,7 \%$ e com o livro foi de $57,9 \%$. Nos cálculos do efeito do uso dos applets, o índice de acerto dos alunos sem eles foi de 47,1\% e com eles, $65,1 \%$.

Por meio do Teste $\mathrm{t}$, foi possível observar que as duas intervenções tiveram efeito significativo, aumentando a média de acerto dos alunos. 


\section{3 Índice de acerto das questões por nível de dificuldade}

As quatro questões classificadas como fáceis tiveram $79,7 \%$ de índice de acerto, enquanto as quatro médias tiveram $48,3 \%$ e as quatro difíceis, $29,9 \%$.

\subsection{Efeito da ordem de uso dos applets}

A média de acerto do grupo que teve a intervenção com o uso dos applets antes do uso do livro foi de $73,96 \%$ e a do grupo que teve o uso dos applets depois do livro, $61,4 \%$.

Pela análise do Teste $\mathrm{t}$, verificou-se que efeito da ordem das intervenções foi significativo $(\mathrm{P}=0,006)$. Dessa forma, é possível afirmar que o uso dos applets para o estudo de áreas de figuras planas para tablets seguido do uso do livro didático é uma alternativa mais eficaz do que usar o livro antes dos applets.

\subsection{Efeito dos applets nos estratos}

\subsubsection{Efeito dos applets com e sem o livro didático}

No cálculo do índice de acerto em cada intervenção, observou-se que o estrato Controle teve um índice de 48,1\%, o Somente applets, 59,9\%, o Somente livro, $42,7 \%$ e o Applets e livro, 67,1\%. A Tabela 3 apresenta a ANOVA entre os efeitos de cada intervenção na média de acerto dos participantes.

Tabela 3 - Efeito das intervenções na média dos participantes

\begin{tabular}{lcc}
\hline Efeitos principais & $\mathrm{F}$ & $\mathrm{P}$ \\
\hline Livro didático & 0,103 & Não significativo \\
Applets & 40,815 & 0,00044 \\
Applets $*$ Livro didático & 5,023 & 0,02556 \\
\hline
\end{tabular}

Observa-se efeito significativo do uso dos Applets e da interação entre Applets e Livro didático, independente da ordem de uso do recurso.

As Tabelas 4 e 5 comparam, respectivamente, os efeitos das interações Applets $\mathrm{x}$ Livro e Livro x Applets. Esclarece-se que o Teste F indica o tamanho da diferença entre as amostras.

Tabela 4 - Interação Applets x Livro*

\begin{tabular}{lcc}
\hline \multirow{2}{*}{ Livro didático } & \multicolumn{2}{c}{ Applets } \\
\cline { 2 - 3 } & Sem applets & Com applets \\
\hline Sem livro & $48,1 \% \mathrm{~B}$ & $59,9 \% \mathrm{~A}$ \\
Com livro & $42,7 \% \mathrm{~B}$ & $67,1 \% \mathrm{~A}$ \\
\hline
\end{tabular}

*Médias seguidas por uma mesma letra, na horizontal, não diferem significativamente entre si $(\mathrm{P}>0,05)$ pelo teste F.

Observa-se que, tanto com o uso do livro quanto sem, o uso dos applets aumentou significativamente as médias dos participantes (Tabela 4). Na Tabela 5, é possível observar que não houve diferença significativa na média dos alunos quando houve o uso dos applets com ou sem livro.

Tabela 5 - Interação Livro x Applets*

\begin{tabular}{lcc}
\hline \multirow{2}{*}{ Applets } & \multicolumn{3}{c}{ Livro didático } \\
\cline { 2 - 3 } & Sem livro & Com livro \\
\hline Sem applets & $48,1 \% \mathrm{~A}$ & $42,7 \% \mathrm{~A}$ \\
Com applets & $59,9 \% \mathrm{~A}$ & $67,1 \% \mathrm{~A}$ \\
\hline *Médias seguidas por uma mesma letra, na horizontal, não diferem significativamente entre si $(\mathrm{P}>0,05)$ \\
pelo teste F.
\end{tabular}

Assim, os applets foram eficazes quando utilizados sozinhos. No entanto, seus efeitos foram potencializados quando o livro foi utilizado (antes ou depois dos applets). 


\subsubsection{Efeito dos applets em questões com níveis de dificuldades diferentes}

A Figura 4 apresenta as médias dos alunos nas questões fáceis, médias e difíceis considerando todas as intervenções. O efeito do uso dos applets foi analisado quando utilizados sozinhos (Somente applets) e antecedidos ou precedidos pelo uso do livro didático (Applets e livro).

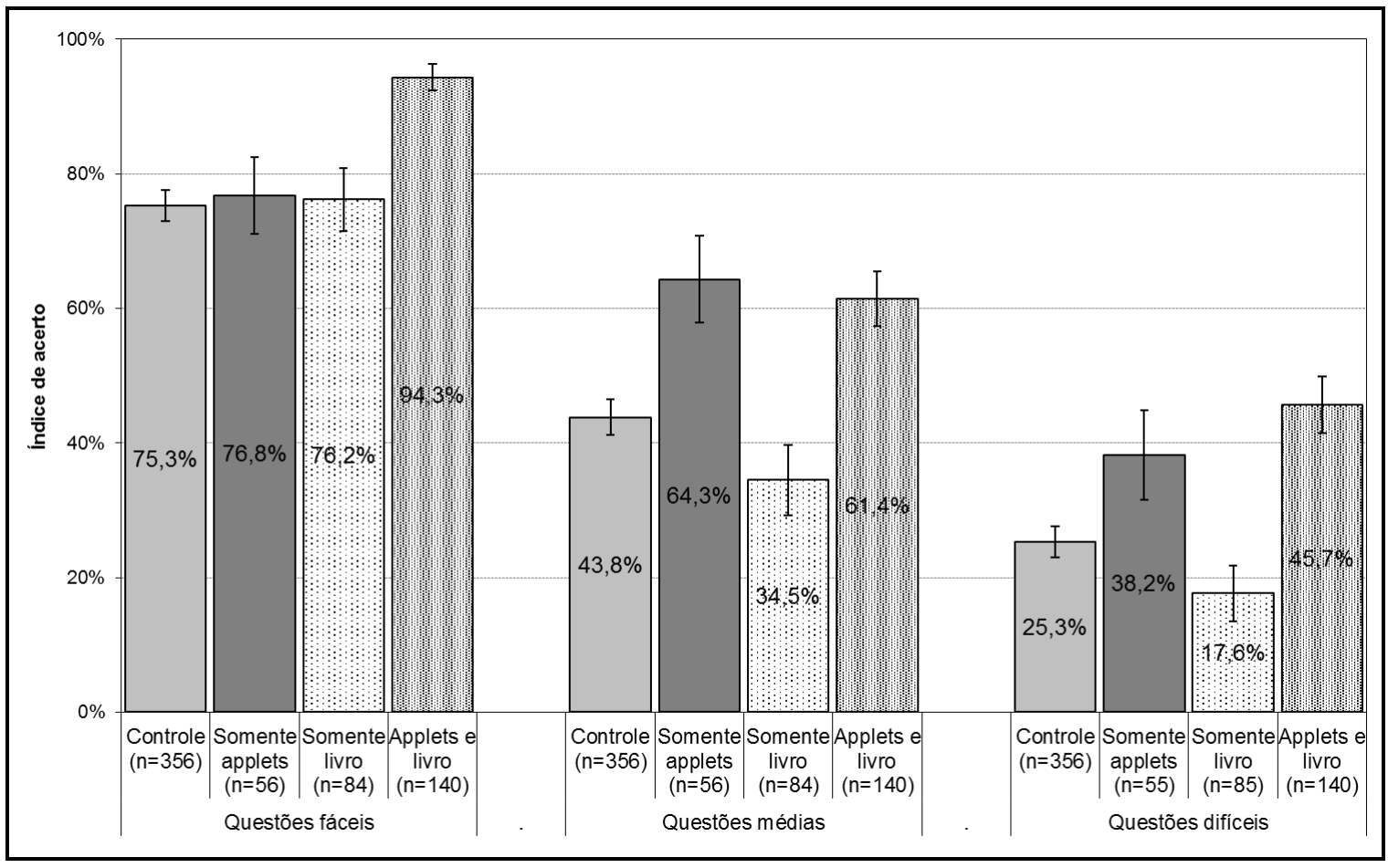

Figura 4 - Médias dos alunos nas questões fáceis, médias e difíceis

As médias referentes a cada intervenção foram comparadas dentro de cada nível de questão utilizando a ANOVA e o Teste de Tukey.

A Tabela 6 mostra a ANOVA entre os efeitos de cada intervenção na média de acerto nas questões fáceis.

Tabela 6 - Efeito das intervenções na média de acerto das questões fáceis

\begin{tabular}{lcc}
\hline Efeitos principais & $\mathrm{F}$ & $\mathrm{P}$ \\
\hline Livro didático & 5,447 & 0,01995 \\
Applets & 6,175 & 0,01325 \\
Applets $*$ Livro didático & 0,693 & 0,03587 \\
\hline
\end{tabular}

Observam-se, pela Tabela 6, efeitos significativos do Livro, dos Applets e da interação entre Applets e Livro didático.

As Tabelas 7 e 8 comparam, respectivamente, os efeitos das interações Livro $\mathrm{x}$ Applets e Applets x Livro, na média de acerto das questões fáceis.

Tabela 7 - Interação Applets x Livro*

\begin{tabular}{lcc}
\hline \multirow{2}{*}{ Livro didático } & \multicolumn{2}{c}{ Applets } \\
\cline { 2 - 3 } & Sem applets & Com applets \\
\hline Sem livro & $75 \% \mathrm{~A}$ & $77 \% \mathrm{~A}$ \\
Com livro & $76 \% \mathrm{~B}$ & $94 \% \mathrm{~A}$ \\
\hline
\end{tabular}

*Médias seguidas por uma mesma letra, na horizontal, não diferem significativamente entre si $(\mathrm{P}>0,05)$ pelo teste F.

$\mathrm{Na}$ Tabela 7, é possível verificar que, sem o uso do livro, os applets não influenciaram significativamente a média de acerto das questões fáceis. No entanto, com o uso do livro, os applets aumentaram significativamente a média de acerto. 
Tabela 8 - Interação Livro x Applets*

\begin{tabular}{lcc}
\hline \multirow{2}{*}{ Applets } & \multicolumn{3}{c}{ Livro didático } \\
\cline { 2 - 3 } & Sem livro & Com livro \\
\hline Sem applets & $75 \% \mathrm{~A}$ & $76 \% \mathrm{~A}$ \\
Com applets & $77 \% \mathrm{~B}$ & $94 \% \mathrm{~A}$ \\
\hline
\end{tabular}

*Médias seguidas por uma mesma letra, na horizontal, não diferem significativamente entre si $(\mathrm{P}>0,05)$ pelo teste F.

Constata-se, pela Tabela 8, que o uso do livro apenas teve efeito significativo quando os applets estavam presentes. Na ausência dos applets, o livro não influenciou a média de acerto das questões fáceis.

Assim, é possível afirmar que, nas questões fáceis, o uso dos applets só teve efeito significativo, quando utilizados antes ou após o uso do livro.

$\mathrm{Na}$ Tabela 9 são apresentados os efeitos de cada uma das intervenções na média de acerto das questões médias e é possível observar efeito significativo do uso do livro e dos applets. Não houve efeito da interação entre Applets e Livro didático.

Tabela 9 - Efeito das intervenções na média de acerto das questões médias

\begin{tabular}{lcc}
\hline Efeitos principais & $\mathrm{F}$ & $\mathrm{P}$ \\
\hline Livro didático & 1,54 & 0,21514 \\
Applets & 23,392 & 0,00004 \\
Applets $*$ Livro didático & 0,1044077 & Não significativa \\
\hline
\end{tabular}

A Tabela 10 mostra os efeitos das intervenções na média de acerto das questões difíceis e observa-se efeito significativo do uso dos applets e da interação Applets e Livro didático.

Tabela 10 - Efeito das intervenções na média de acerto das questões difíceis*

\begin{tabular}{lcc}
\hline Efeitos principais & $\mathrm{F}$ & $\mathrm{P}$ \\
\hline Livro didático & $<0,001$ & Não significativo \\
Applets & 20,881 & 0,00004 \\
Applets $*$ Livro didático & 2,862 & 0,09124 \\
\hline
\end{tabular}

Assim, nas questões médias e difíceis, o uso dos applets teve efeito significativo na média de acertos dos participantes.

\section{Considerações Finais}

As análises dos dados permitiram identificar que usar os applets para o estudo de áreas de figuras planas para tablets antes de usar o livro didático foi uma alternativa mais eficaz do que na ordem contrária. Além disso, de forma geral, os applets se mostraram significativamente eficazes tanto com o uso do livro, quanto sem o uso dele.

$\mathrm{Na}$ análise da eficácia do uso dos applets no índice de acerto das questões com diferentes níveis de dificuldades, concluiu-se que nas questões fáceis, o uso dos applets só foi eficaz quando houve o uso do livro. Já nas questões médias e difíceis, o uso dos applets teve efeito significativo.

A eficácia dos applets GeoGebra pode se dever ao fato de que eles permitem obter uma visualização dinâmica de vários elementos, proporcionando aos alunos uma variedade de experiências ligadas à aprendizagem de áreas de figuras planas. Os applets permitiram que os alunos fizessem experimentações e investigações, a fim de deduzir as fórmulas para o cálculo das áreas.

Destaca-se que não houve a pretensão de comparar o uso dos applets com o uso do livro didático nem com outros recursos digitais. Assim, o estudo não permitiu tirar conclusões sobre a eficácia dos applets GeoGebra para o estudo de áreas de figuras planas desenvolvidos em relação a outras abordagens.

Como sugestões para a continuidade do trabalho desenvolvido apontam-se: i) avaliar a eficácia de applets GeoGebra para tablets com alunos que possuem alguma 
necessidade intelectual, como, por exemplo, déficit de atenção; e, ii) investigar a preferência de professores quanto ao uso dos applets GeoGebra em tablets e em computadores desktops/notebooks.

Considerando-se a escassez de estudos quantitativos e conclusivos quanto a eficácia de aplicativos de Matemática para tablets e a não identificação de estudos sobre applets GeoGebra em tablets, destaca-se a importância do trabalho aqui descrito.

\section{Referências}

ADAMS, L.; CHUNG, C. J. The Effect of an iPad for Every Student. In: SOCIETY FOR INFORMATION TECHNOLOGY AND TEACHER EDUCATION INTERNATIONAL CONFERENCE, 2013, Chesapeake. Proceedings. Chesapeake, VA: AACE, 2013. p. 3569 - 3572.

AGOSTINHO, S. et al. Giving Learning a helping hand: Finger tracing of temperature graphs on an iPad. Educational Psychology Review, v. 27, n. 3, p.427-443, 5 jun. 2015.

AMIRNUDIN, M. T. M.; SULAIMAN, H. Exploring the use of tablet technology as a teaching tool at Kolej Matrikulasi Perak. In: NATIONAL SYMPOSIUM ON MATHEMATICAL SCIENCES: RESEARCH IN MATHEMATICAL SCIENCES, 20., 2013, Putrajaya. Proceedings. Putrajaya: AIP Publishing, 2013. p. 590 $-595$.

BARCELOS et al. Uso Educacional de Tablets: estudo de caso na formação inicial de professores de Matemática. Revista Novas Tecnologias na Educação, v. 11, n.1, p. 110, jul. 2013.

HAVELKOVÁ, V. GeoGebra in Teaching Linear Algebra. In: INTERNATIONAL CONFERENCE ON E-LEARNING, 12., 2013, Valbonne. Proceedings. Valbonne: Academic Conferences and Publishing International Limited Reading, 2013. p. 581 589.

PEREIRA, B. T.; PEIXE, B. C. S.; STARON, L. Avaliar a Eficiência e Eficácia da Gestão Escolar Integral no Processo Ensino-Aprendizagem: Estudos de Casos nas Escolas Estaduais da Região de Campo Largo. In: PEIXE, B. C. S. et al. (Org.). Formulação e Gestão de Políticas Públicas no Paraná: Reflexões, Experiências e Contribuições. Curitiba: Imprensa Universitária da Universidade Federal do Paraná, 2010. p. 17-31.

PITCHFORD, N. J. Development of early mathematical skills with a tablet intervention: a randomized control trial in Malawi. Frontiers in Psychology, Lausana, v. 6 , n. 485 , p.1-2, 23 abr. 2015.

RICONSCENTE, M. M. Results from a controlled study of the iPad fractions game Motion Math. Games and Culture, v. 8, n. 4, p.186-214, 1 jul. 2013.

ZHANG, M. et al. Using Math Apps for Improving Student Learning: an Exploratory Study in an Inclusive Fourth Grade Classroom. TechTrends, v. 59, n. 2, p.32-39, 28 jan. 2015. 